\title{
A biobank to support HIV malignancy research for sub-Saharan Africa
}

\author{
J W Schneider, ${ }^{1}$ MB ChB, MMed, FCPath; M Sanderson, ${ }^{1} \mathrm{PhD}$; D Geiger, ${ }^{1}$ MSc; M Nokta, ${ }^{2} \mathrm{MD}, \mathrm{PhD} ; \mathbf{S}$ Silver, ${ }^{3} \mathrm{DA}$ \\ ${ }^{1}$ Division of Anatomical Pathology, National Health Laboratory Service, Tygerberg Academic Hospital and Faculty of Medicine and Health \\ Sciences, Stellenbosch University, Tygerberg, Cape Town, South Africa \\ ${ }^{2}$ Office of HIV and AIDS Malignancy, AIDS Cancer Clinical Program, National Cancer Institute, National Institutes of Health, Bethesda, \\ Md, USA \\ ${ }_{3}^{3}$ Department of Microbiology, Immunology, and Tropical Medicine, George Washington University School of Medicine and Health Sciences, \\ Washington, DC, USA
}

Corresponding author: JW Schneider (jws2@sun.ac.za)

\begin{abstract}
Sub-Saharan Africa has one of the highest incidences of infection with HIV globally, but more people in this region are living longer owing to increased access to antiretroviral therapy. However, along with increased care and treatment, this population is expected to have an increase in HIV-associated cancers, as is being seen in the USA and other developed countries. To support translational research in HIV-associated cancers, Stellenbosch University in Cape Town, South Africa, was funded to house the state-of-the-art AIDS and Cancer Specimen Resource Sub-Saharan Africa Regional Biorepository (SSA RBR) to proactively obtain, manage and process biospecimens and associated clinical data representing both AIDS-defining and non-AIDS-defining cancers for research. The SSA RBR furthermore functions as the biorepository for AIDS Malignancy Consortium sub-Saharan clinical trial activities in this region. Although the site had much experience with cryopreservation and storage of specimens, capacity building revolving around operations under International Society for Environmental and Biological Resources/National Cancer Institute best practices took place in such areas as custodianship v. ownership, data sharing and facilities management. The process from selection until launch took 14 months.
\end{abstract}

S Afr Med J 2016;106(9):867-869. DOI:10.7196/SAMJ.2016.v106i9.10891

The generation of quality data in biomedical research is dependent on utilisation of high-quality specimens. Although scientific publications overflow with research results and mounds of data, not necessarily all bring useful results. As stated by the US National Cancer Institute (NCI), the lack of standardised high-quality biospecimens is the cause of the lack in progress in cancer research. ${ }^{[1]}$ High-quality research material and matching clinical data are also essential to comply with growing international requirements from regulatory authorities and funding agencies that biospecimens should be handled according to best practices. The latter include appropriately qualified staff who operate in a facility that can demonstrate high-quality management of research specimens pertaining to conditions of sample collection, preparation, storage and transportation, secure record-keeping, processes that regulate access to and utilisation of biospecimens, long-term sustainability strategies, and compliance with ethical and legal requirements. ${ }^{[2]}$

After collection, specimens for research are usually stored until they are needed. Ubiquitous in most research laboratories is the $-80^{\circ} \mathrm{C}$ freezer, loaded with saved specimens from one or more investigators. However, in view of the globally expanding drive for population-based and evidence-based research, access to and utilisation of biospecimens in this familiar environment are growing to the scale of requiring a biobank/biorepository.

A biobank is defined as an entity that acquires, preserves, develops and distributes biological specimens along with information, technology, intellectual property, and standards for the advancement and application of scientific knowledge. ${ }^{[3]}$ Biobanks operated under best practices are important partners to translational health researchers, as they ensure high-quality specimens for their studies and employ functions and policies to ensure not only sound ethics but also quality through technical standards and operational

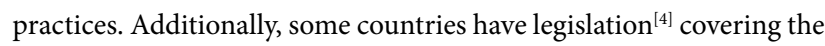
collection and use of human specimens in research. Governmental agencies and professional organisations such as the International Society for Environmental and Biological Resources (ISBER) produce standards to set a benchmark for quality. ${ }^{[5,6]}$

Variations in biobanks exist. Biobanks can be devoted to the collection of human specimens (e.g. tissues, DNA/RNA, blood, sperm) or animal, plant (e.g. seeds), microbial and environmental specimens. The biobank's mission determines its scope and size. For example, biobanks related to human research can support basic science, translational science or clinical research. Biobanks vary in their size and in the type of specimens they store, and in the case of human biobanks, according to whether the specimens are from healthy individuals or from individuals with disease. Specimens can be banked from a single research project or represent diverse research areas.

The structure of a biobank can take numerous forms. If a bank is traditional, it holds specimens and data that are stored centrally. A procurement biobank collects specimens to meet a specific need, and may only have a limited data set. Virtual biobanks have multiple collection locations but a central location for the storage of data. A fully virtual/distribution bank has data and multiple collections and storage sites. ${ }^{[7]}$

The AIDS and Cancer Specimen Resource (ACSR), in existence since 1994, operates under a cooperative agreement between the $\mathrm{NCI}$ and various institutions. Its mandate is to collect and preserve tissues and biological fluids along with clinical data from patients with HIV-related malignant tumours and normal controls, which will be dispersed to qualified investigators. ${ }^{[8,9]}$ The ACSR recently expanded its scope to include a regional biorepository to serve sub-Saharan 
Africa (SSA RBR), located in the Division of Anatomical Pathology, National Health Laboratory Service (NHLS), Tygerberg Academic Hospital and Faculty of Medicine and Health Sciences, Stellenbosch University (Cape Town, South Africa (SA)). Under the auspices of the ACSR, the SSA RBR aims to proactively obtain, manage and process biospecimens (tissues, body fluids and derivatives, such as DNA and RNA) representing both AIDS-defining cancers (i.e. Kaposi's sarcoma, non-Hodgkin's lymphoma, cervical cancer) and non-AIDS-defining cancers (e.g. lung cancer, breast cancer, colon cancer) and associated clinical data (e.g. date of diagnosis, site of biopsy, antiretroviral and cancer therapies, CD4 counts). Ethics approval was secured and regulatory aspects ensured for specimen movement within the continent. Additional aims include the provision of information and training related to biobanking for African researchers and scientists, and the establishment of a community advisory board and community outreach portal to educate and to inform the public about the benefits and implications of biobanking for the health of African populations.

The SSA RBR also hosts the AIDS Malignancy Consortium (AMC) biorepository for support of its clinical trial activities in SSA. The $\mathrm{AMC}^{[10]}$ supports innovative clinical trials for optimising treatment and prevention of AIDS-related cancers. The AMC was established in 1995 to perform domestic studies in the USA. In the past 5 years the AMC widened its scope to conduct trials in international settings, particularly in SSA. The SSA RBR supports the biorepository requirements for AMC clinical trials performed on the African continent. This function involves receiving and storage of specimens under controlled conditions before sending them to specified core laboratories in Africa and globally. The process of establishing a biobank to fulfil both the ACSR and AMC mandates is described.

\section{Developing the biobanking concept}

Acceptance of biobanks to support research in SA and other African countries cannot be automatically assumed. One must be sensitive to past infringements of ethicoregulatory standards by international researchers, where many Africans repeatedly witnessed biological specimens leaving the continent to other countries ${ }^{[1]}$ for research with no clear benefit to the African communities.

Scientifically and technologically, establishing the biobank was not a concern, but various concepts covered by the NCI and ISBER best practices were new and required adequate time to be adopted. Concomitantly, it was essential that this new site would relate to the ACSR mission 'to acquire, store, and equitably distribute tumor tissues, biological fluids, and other biospecimens and associated clinical information from patients with HIV-associated malignancies to the scientific research community-at-large. The concept and scope of the biobank were easily accepted, particularly since the prevalence of HIV/AIDS in SA is one of the highest in the world.

The biobank's location in the immediate proximity of the laboratories of the Division of Anatomical Pathology at Tygerberg Academic Hospital facilitated the identification of qualified individuals who supported the establishment of the RBR infrastructure, and adoption of the manual of operations, standard operating procedures and bioinformatics platform of the ACSR. Besides discussions related to laboratory procedures for biobanks, and of the importance of preanalytical variables and controlling for them on research specimens, ${ }^{[12,13]}$ selected best practices identified for more in-depth exploration are presented below.

\section{Organisation, management and governance}

Stellenbosch University, the site chosen for the new AMC/ACSR SSA biorepository, already had in-house repositories holding research study specimens for storage and distribution. In the existing scenarios, all specimens in the respective repositories were obtained as part of approved research projects and controlled and managed by the respective study investigators. Stellenbosch University already had regulatory offices in place to handle entry and exit permits and material transfer agreements; consequently, the mission of the AMC biorepository fitted aptly with other established projects. In addition, most of the African countries involved with AMC trials were already research partners with Stellenbosch University.

What was new, however, was the ACSR mission of procuring specimens to be used by investigators at that institution as well as by individuals from outside the institution, outside SA, and even outside Africa. At this point, in-depth discussions occurred regarding: (i) the ACSR mission; (ii) the functions of the ACSR regional biorepositories; (iii) the governance of the ACSR; (iv) how the new SAA RBR would fit into the present ACSR; $(v)$ the mechanism for investigators to request specimens; ( $v i$ ) how the requests were reviewed; and (vii) distribution of biospecimens and information sets.

A specific challenge emerged in view of the mandate by the NCI to ensure that findings derived from its inventory would benefit Africa and assist in building capacity for research. The SSA RBR would be unique within the ACSR in that investigators desiring specimens from its collection would need to be African or collaborating with an African researcher. This mission required various discussions between individuals ranging from other ACSR directors and members of the Health Research Ethics Committee (HREC) to people working with regulatory issues, e.g. entry/exit permits and material transfer agreements.

The SSA RBR complies with the South African National Health Act 61 of 2003 and adheres to the Ethics in Health Research Principles, Processes and Structures, as published in 2015 by the SA National Department of Health. Following extensive discussions with relevant authorities, the SSA RBR obtained permission from the Department of Health in the Western Cape Province and support from the NHLS. The SSA RBR operates according to inspection criteria as determined and monitored by the inspector of anatomy from the Western Cape Department of Health. It conforms and contributes to evolving ethicoregulatory detail and policies on biobanking and biospecimen utilisation in SA, and will pursue similar involvement in other SSA countries.

\section{Custodianship v. ownership}

The ACSR's mandate to collect specimens and make them available to any African or collaborating researcher approved through peer review was a new concept, and one requiring some discussion. A major discussion with the HREC revolved around Stellenbosch University's role as the custodian of the specimen (i.e. the caretaker of the specimen that ensures the privacy of the donor, the integrity of the specimen and its associated data, and that use of both conforms to informed consent) rather than its role as owner of the specimen (that 'donates' its specimen to research and can retract that permission at any time). Also needing clarification was concern regarding the return of data from successful investigators who performed research on the SSA RBR specimens. The ACSR encourages successful recipients of ACSR samples to share their findings with the ACSR to enrich the remaining specimens' value. However, as the specimens are only used for research and not clinical decisions, the donor of a specimen is never notified about investigative findings; rather, investigators share and disseminate findings through published articles and presentations of research findings at scientific meetings, acknowledging the ACSR. 


\section{Community awareness and involvement}

The SSA RBR promotes ethical and equitable biobanking policies for community and public education regarding biobanking and its role and value in improving the health of people on the African continent. For this purpose, the SSA RBR has developed a marketing and promotion strategy that is based on established ACSR principles but specifically directed at various SA target groups. Briefly, the strategy includes regular sharing of information pertaining to the SSA RBR with clinicians, health authorities, the general public and selected SA and African researchers; close collaboration with established researchers with African involvement to explore recruitment of specimens from clinical trial patients, involvement of selected societies that are active in the field of HIV and cancer, co-operation with community advisory boards, and regular presentations at regional congresses are further components of the marketing and promotion strategy.

A further responsibility taken on by the ACSR and its role of custodianship is the reporting of data found by investigators utilising ACSR specimens. The ACSR lists publications by inventory users on its website (http://acsr.ucsf.edu). Specific to the SSA RBR is sharing of information and results through the SSA RBR Community Advisory Board. This group of individuals comprises lay people working within the fields of or living with either HIV or cancer. The SSA RBR will periodically present, in lay language, summaries of the proposed research and the findings from research performed on the specimens to the group. This is a work in progress, with emphasis on the appropriate level of presentation of information to facilitate comprehension.

\section{Discussion}

The interval from award to launch of the SSA RBR was 14 months. The approach involved taking small steps at a time, with different levels of involvement from the administration, the faculty and the principal staff as necessary. Long-range plans include bringing in specimens from other sites in the Western and Eastern Cape provinces, as well as the rest of SA and eventually SSA. Targeted discussions were held between the biorepository leadership and the stakeholders, ethics officers and regulatory officials at various steps of biobank development.

The biobank currently has full HREC approval from the Faculty of Medicine and Health Sciences at Stellenbosch University and Tygerberg Academic Hospital, the tertiary academic hospital of Stellenbosch University. Meetings with most of the clinical departments pertinent to HIV malignancies have been held. The SSA RBR nurses have been trained as research nurses, and they request informed consent from patients identified by clinicians. The inventory is growing, and the first request for biospecimens (by an African investigator) has occurred. All requests for biospecimens will be vetted by an independent research evaluation and decision panel composed of experts identified by the NCI, including one or two representatives from Africa. Final approval for dispersal of ACSR biospecimens lies with the ACSR executive committee, in which the director of the SSA RBR is a member.

The SSA RBR director has begun outreach to other hospitals in Cape Town and the surrounding areas to raise the awareness of clinicians, scientists and junior investigators of the existence of the biobank. This effort involves meeting with clinical heads and pathologists from each hospital, and presenting the ACSR mission and the operation logistics envisioned for collaborating sites. In this scenario, the SSA RBR will work with the site to produce a standard operating procedure for informed consent and collection and transport of the specimen and data to the SSA RBR. Appropriate support will be supplied in the form of personnel needed for consent and collection, a pathologist for verification of diagnosis, and transport. These actions go towards the long-range plan for the SSA RBR to eventually house inventory from all of SSA.

\section{Conclusion}

The SSA RBR is a resource to benefit researchers, patients and communities in Africa. It offers numerous advantages, including operation under accepted best practices in biorepository management, with quality management in governance, personnel, operations and safety. It furthermore offers an opportunity to monitor trends in biospecimen research from an African perspective. Through collaboration with other institutions in SSA, the SSA RBR creates an opportunity to develop unique specimen collections and clinical data sets to promote multi-institutional collaborative research in the field of HIV-related cancers. The SSA RBR serves as a site for future National Institutes of Health/NCI cancer activities in Africa.

1. National Cancer Institute. Biospecimen and biorepository research branch website http // biospecimens.cancer.gov/about/overview.asp (accessed 8 July 2015).

2. Somiari SB, Somiari RI. The future of biobanking: A conceptual look at how biobanks can respond to the growing human biospecimen needs of researchers. Adv Exp Med Biol 2015;864:11-27. to the growing human biospecime
DOI:10.1007/978-3-319-20579-3_2

3. Cunningham A, Martinez RM, Hernandez DR, et al. The microbe farms: Microbial biorepositories in clinical microbiology. Clinical Microbiology Newsletter 2014;36(6):41-48. DOI:10.1016/j. clinmicnews.2014.02.003

4. García-Merino IM, Consuegra I, Jiménez JL, et al. Specific legislation on biobanks in Spain. Biopreserv Biobank 2015;13(3):207-211. DOI:10.1089/bio.2014.0070

5. NCI Best Practices for Biospecimen Resources. Table of contents. 2011. http://biospecimens.cancer gov/bestpractices/toc/ (accessed 24 June 2015).

6. Campbell LD, Betsou F, Garcia DL, et al. Development of the ISBER Best Practices for Repositories: Collection, storage, retrieval and distribution of biological materials for research. Biopreserv Biobank 2012;10(2):232-233. DOI:10.1089/bio.2012.1025

7. Reijs BLR, Teunissen CE, Goncharenko N, et al. The central biobank and virtual biobank of BIOMARKAPD: A resource for studies on neurodegenerative diseases. Front Neurol 2015;15(6):216 DOI:10.3389/fneur.2015.00216

8. Ayers LW, Silver S, Orenstein JM, et al. The AIDS and cancer specimen resource. Methods Mol Biol 2011;675:193-203. DOI:10.1007/978-1-59745-423-0_8

Ayers LW, Silver S, McGrath MS, et al. The AIDS and cancer specimen resource: Role in HIV/AIDS . Ayers LW, Silver S, McGrath MS, et al. The AIDS and cancer specimen resource:
scientific discovery. Infect Agent Cancer 2007;2:7. DOI:10.1186/1750-9378-2-7

10. AIDS Malignancy Consortium. http://pub.emmes.com/study/amc/public/index.htm (accessed 5 March 2016).

11. Staunton C, Moodley K. Challenges in biobank governance in Sub-Saharan Africa. BMC Med Ethics 2013;14:35. DOI:10.1186/1472-6939-14-35

12. Lehmann S, Guadagni F, Moore H, et al. International Society for Biological and Environmental Repositories ISBER Working Group on Biospecimen Science F. Standard preanalytical coding for biospecimens: Review and implementation of the Sample PREanalytical Code (SPREC). Biopreserv Biobank 2012;10(4):366-374. DOI:10.1089/bio.2012.0012

13. Ellervik C, Vaught J. Preanalytical variables affecting the integrity of human biospecimens in biobanking. Clin Chem 2015;61(7):914-934. DOI:10.1373/clinchem.2014.228783 